\title{
A tidal tale: detection of several stellar streams in the environment of NGC 1052
}

\author{
Oliver Müller ${ }^{1}$, R. Michael Rich ${ }^{2}$, Javier Román ${ }^{3,4}$, Mustafa K. Yıldız ${ }^{1,5,6}$, Michal Bílek ${ }^{1}$, Pierre-Alain Duc ${ }^{1}$, \\ Jérémy Fensch ${ }^{7}$, Ignacio Trujillo ${ }^{3,4}$, and Andreas $\mathrm{Koch}^{8}$ \\ ${ }^{1}$ Université de Strasbourg, Observatoire Astronomique de Strasbourg (ObAS), CNRS UMR 7550, Strasbourg, France \\ e-mail: oliver.muller@astro.unistra.fr \\ 2 Dept. of Physics and Astronomy, UCLA, Los Angeles, CA 90095-1547, USA \\ 3 Instituto de Astrofísica de Canarias (IAC), La Laguna 38205, Spain \\ 4 Departamento de Astrofísica, Universidad de La Laguna (ULL), 38200 La Laguna, Spain \\ 5 Astronomy and Space Sciences Department, Science Faculty, Erciyes University, Kayseri 38039, Turkey \\ ${ }^{6}$ Erciyes University, Astronomy and Space Sciences Observatory Applied and Research Center (UZAYBİMER), \\ 38039 Kayseri, Turkey \\ 7 European Southern Observatory, Karl-Schwarzschild Strasse 2, 85748 Garching, Germany \\ 8 Astronomisches Rechen-Institut, Zentrum für Astronomie der Universität Heidelberg, Mönchhofstr. 12-14, \\ 69120 Heidelberg, Germany
}

Received 13 March 2019 / Accepted 25 March 2019

\begin{abstract}
The possible existence of two dark-matter-free galaxies (NGC 1052-DF2 and NGC 1052-DF4) in the field of the early-type galaxy NGC 1052 presents a challenge to theories of dwarf galaxy formation according to the current cosmological paradigm. We carried out a search for signatures of past interactions connected to the putative hosts of NGC 1052-DF2 and NGC 1052-DF4 using a very deep $L$-band image obtained with the $0.7 \mathrm{~m}$ Jeanne Rich telescope that reach a surface brightness limit of $28.5 \mathrm{mag} \mathrm{arcsec}^{-2}$ in the $r$ band. We found several low-surface brightness features, possibly consistent with an ongoing merger history in this group. We find a tidal interaction between NGC 1052 and NGC 1047, confirming a physical association. Furthermore, we find a stellar loop around NGC 1052 in the direction of NGC 1042 and a stellar stream pointing in the direction of NGC 1052-DF2, but they are not directly connected. We find no evidence for a recent tidal interaction for NGC 1052-DF2 and NGC 1052-DF4. No LSB features have been uncovered around the spiral galaxy NGC 1042, which leaves the association (physical or projected) between NGC 1052 and NGC 1042 ambiguous, although they have similar radial velocities. Their association will only be established when accurate distances to both objects have been measured.
\end{abstract}

Key words. galaxies: groups: individual: NGC 1052 - galaxies: halos

\section{Introduction}

The discovery of two apparently dark-matter-free dwarf galaxies in the field of the early-type galaxy NGC 1052 has sparked strong interest with numerous follow-up studies (e.g., van Dokkum et al. 2018a,b; Trujillo et al. 2019; Martin et al. 2018; Kroupa et al. 2018; Famaey et al. 2018; Emsellem et al. 2019; Fensch et al. 2019; Müller et al. 2019; Nusser 2019; Laporte et al. 2019). These two galaxies, NGC 1052-DF2 ${ }^{1}$ (van Dokkum et al. 2018a) and NGC 1052-DF4 (van Dokkum et al. 2019), are very extended $\left(r_{\text {eff }}>1500 \mathrm{pc}\right)$ and very $\operatorname{dim}\left(m u_{\mathrm{eff}, V}>25 \mathrm{mag} \operatorname{arcsec}^{-2}\right)$ at a putative distance of NGC 1052, but their distances are still a matter of active debate (van Dokkum et al. 2018b; Trujillo et al. 2019). Objects of this size and luminosity were first discovered in the Virgo cluster by Sandage \& Binggeli (1984), and recent

1 Originally, this galaxy was discovered by Fosbury et al. (1978) and has also been reported by Karachentsev et al. (2000), who called it [KKS2000] 04, but here we follow the naming convention in recent literature. surveys of galaxies with low surface brightness (LSB) identified them in different galactic environments (e.g., Mihos et al. 2015; Merritt et al. 2016; van der Burg et al. 2016; Ordenes-Briceño et al. 2016; Wittmann et al. 2017; Venhola et al. 2017; Román \& Trujillo 2017a,b; Karachentsev et al. 2015; Eigenthaler et al. 2018; Müller et al. 2018a; Greco et al. 2018; Mancera Piña et al. 2019). This class of galaxies has been rebranded ultra-diffuse galaxies (UDG; van Dokkum et al. 2015).

The apparent lack of dark matter in NGC 1052-DF2 and NGC 1052-DF4 makes these objects interesting and has raised discussions about their origin. A primordial dwarf galaxy should always contain a dynamically significant portion of dark matter, see, for instance, the dual dwarf theorem in Kroupa (2012) and its implications. This means that the possible absence of dark matter in these galaxies is a direct contradiction of the current cosmological paradigm. Several scenarios have been suggested, for example, that they were tidally stripped from their dark matter by a close passage to NGC 1052 (Ogiya 2018), or that they are the direct results of a tidal interaction, that is, tidal dwarf galaxies (TDG; Duc 2012; Duc et al. 2014). Mergers 
producing such TDGs are expected to leave observable signatures in the form of several streams and shells around NGC 1052 (and/or the other massive galaxies in the field) that can potentially be detected by deep imaging for several gigayears after the interaction.

NGC 1052 is an active radio galaxy consisting of two radio sources, a compact and an extended source in the form of a twosided radio jet (Tingay et al. 2003; Kadler et al. 2004). Pierce et al. (2005) studied the recent history of NGC 1052 and argued that the young central starburst had to start from gas that is enriched in $\alpha$ elements. Gas like this is usually found in massive spirals. This young central starburst has an age estimate of $\sim 1 \mathrm{Gyr}$, coinciding with the estimated age of the accretion of the H I clouds found around NGC 1052 (van Gorkom et al. 1986). Its distance is estimated with surface brightness fluctuations (SBF) and measured to be $D=19 \mathrm{Mpc}$ (Tully et al. 2013). Another giant galaxy in the same field is NGC 1042. This is a spiral galaxy separated by $15^{\prime}$ from NGC 1052 to its southeast. Various distance estimates have placed NGC 1042 between 4 and $20 \mathrm{Mpc}$ (Tully et al. 1992, 2008; Theureau et al. 2007; Luo et al. 2016). A reconstruction of the velocity field of the galaxies in the local universe places NGC 1042 at a distance of $13 \mathrm{Mpc}$ (Theureau et al. 2007), which would mean that NGC 1052 and NGC 1042 are companions in projection only. However, they have similar velocities: $v=1376 \mathrm{~km} \mathrm{~s}^{-1}$ for NGC 1042 (Adelman-McCarthy 2009) and $v=1510 \mathrm{~km} \mathrm{~s}^{-1}$ for NGC 1052 (Denicoló et al. 2005). Furthermore, in the extended field around NGC 1052 , there appears to be a plethora of giant galaxies that share the same velocity (Garcia 1993; see also Trujillo et al. 2019).

In this work, we raise the question whether NGC 1052-DF2 and NGC 1052-DF4 are physically associated with the giant galaxies, and also whether tidal interactions can be observed between the galaxies. We have acquired deep images to recover the low-surface brightness features of the field around NGC 1052 and probe the signatures of tidal features.

\section{Observations and data processing}

As part of the Halos and Environments of Nearby Galaxies (HERON) survey (Rich et al. 2017, 2018), we have acquired deep images with the $0.7 \mathrm{~m}$ Jeanne Rich telescope (Brosch et al. 2015; Rich et al. 2017) located at Lockwood Valley (CA, USA) between October and November 2018. The camera has a pixel scale of 1.1 arcsec and a field of view of $\sim 1$ square degree, allowing us to cover NGC 1052, NGC 1042, NGC 1052-DF2, and NGC 1052-DF4 in a single image field. In total, we secured $245 \times 300 \mathrm{~s}$ exposures, giving a total exposure of $20.4 \mathrm{~h}$. The observations were conducted at an airmass between 1.4 and 1.8. All observations were taken under photometric conditions when the Moon was below the horizon. During the observations the sky brightness was between 21.7 and 22.0 mag arcsec $^{-2}$ ( $V$ band). The reduction process was carried out by correcting bias and flat-fielding. For the creation of the flat, we masked the science images using Noisechisel (Akhlaghi \& Ichikawa 2015) and combined them. Because there were gradients, we used Noisechisel to obtain the sky of each image, which was subtracted from the images that were before corrected for bias and flat-fielding. Astrometry was obtained through the scamp package (Bertin 2006). Finally, we combined all exposures with a $3 \sigma$ resistant mean.

The zero-point was derived using APASS (Henden \& Munari 2014). To transform from our instrumental luminance $L$ magnitudes to the commonly used SDSS $r$ band, we applied a linear transformation using 25 standard stars from the APASS catalog. We determined the surface brightness limits by measuring the standard deviation of blank pixels (pixels with no sources) in $10 \times 10$ arcsec boxes on the luminance $L$ image. The $3 \sigma$ standard deviation, transformed to the $r$-band using the linear photometric transformation, is $\mu_{\text {lim, } r}=28.5 \mathrm{mag} \mathrm{arcsec}^{-2}$. This limit is comparable to the depth reached by other telescopes of modest aperture size (Martínez-Delgado et al. 2010; Karachentsev et al. 2015; Javanmardi et al. 2016; Henkel et al. 2017).

\section{Low surface brightness features}

Several LSB features stand out in the field around NGC 1052 in Fig. 1. We modeled NGC 1052 using the ellipse algorithm in IRAF (Tody 1986) after removing the faint objects and masking bright stars together with the companion galaxies. The modelsubtracted image is presented in Fig. 2. Most prominently, a narrow stream is emitted from NGC 1052 into the vicinity of NGC 1052-DF2. We refer to this as Stream 1. A loop is visible to the southwest (loop SW), close to NGC 1042, but apparently disconnected from it. The tidal tail as seen in $\mathrm{HI}$ around NGC 1052 extends toward NGC 1047 (see Fig. 2). NGC 1047 shows a clear tidal distortion or extension to the northwest, which is on the opposite side from NGC 1052. NGC 1047 also shows tidal features toward NGC 1052 that might be a bridge. The outer isophotes of NGC 1047 appear to be boxy. We also note an arc feature to the northwest of NGC 1052. While we find multiple tidal features around NGC 1052, no convincing features are visible around NGC 1042.

Stream 1 is a striking feature. It appears as a straight line coming from NGC 1052 in the direction of NGC 1052-DF2. It stops 100 arcsec before the center of NGC 1052-DF2. The residual image of NGC 1052 shows that Stream 1 extends toward the center of the bright elliptical galaxy.

To study the LSB features around NGC 1052-DF2 and NGC 1052-DF4, we modeled and subtracted the two galaxies from the images (Fig. 3). After the subtraction, no remaining features were left. While the position angle of NGC 1052-DF2 changes with respect to the radius, NGC 1052-DF4 shows no sign of this.

\section{Discussion}

NGC 1052 resides in an environment where multiple interactions occurred in the past and are still ongoing. The LSB features between NGC 1052 and NGC 1047 and the lopsided LSB feature on the far side of NGC 1047 undoubtedly show that interaction is ongoing between NGC 1052 and NGC 1047; they are therefore at the same distance. The boxy appearance of the outer isophotes of NGC 1047 further indicates tidal disturbance. NGC 1052 also contains an irregularly shaped distribution of H I (van Gorkom et al. 1986), with what appears to be a tidal arm extending toward the southwest. van Gorkom et al. (1986) argued that H I has been acquired 1 Gyr ago. In addition, Tingay et al. (2003) argued that NGC 1052 is a restarted radio source. The extended radio source is likely much older than the compact radio source at the nucleus, and these sources could have been created during different events.

The relative position of Stream 1 and NGC 1052-DF2 may suggest that the galaxy is a TDG formed at the tip of a tidal arm (Bournaud \& Duc 2006). NGC 1052-DF2 and NGC 1052DF4 indeed have the structural properties of old TDGs (central surface brightness, large effective radius, lack of dark matter content) (Duc et al. 2014). While the hypothesis of a 


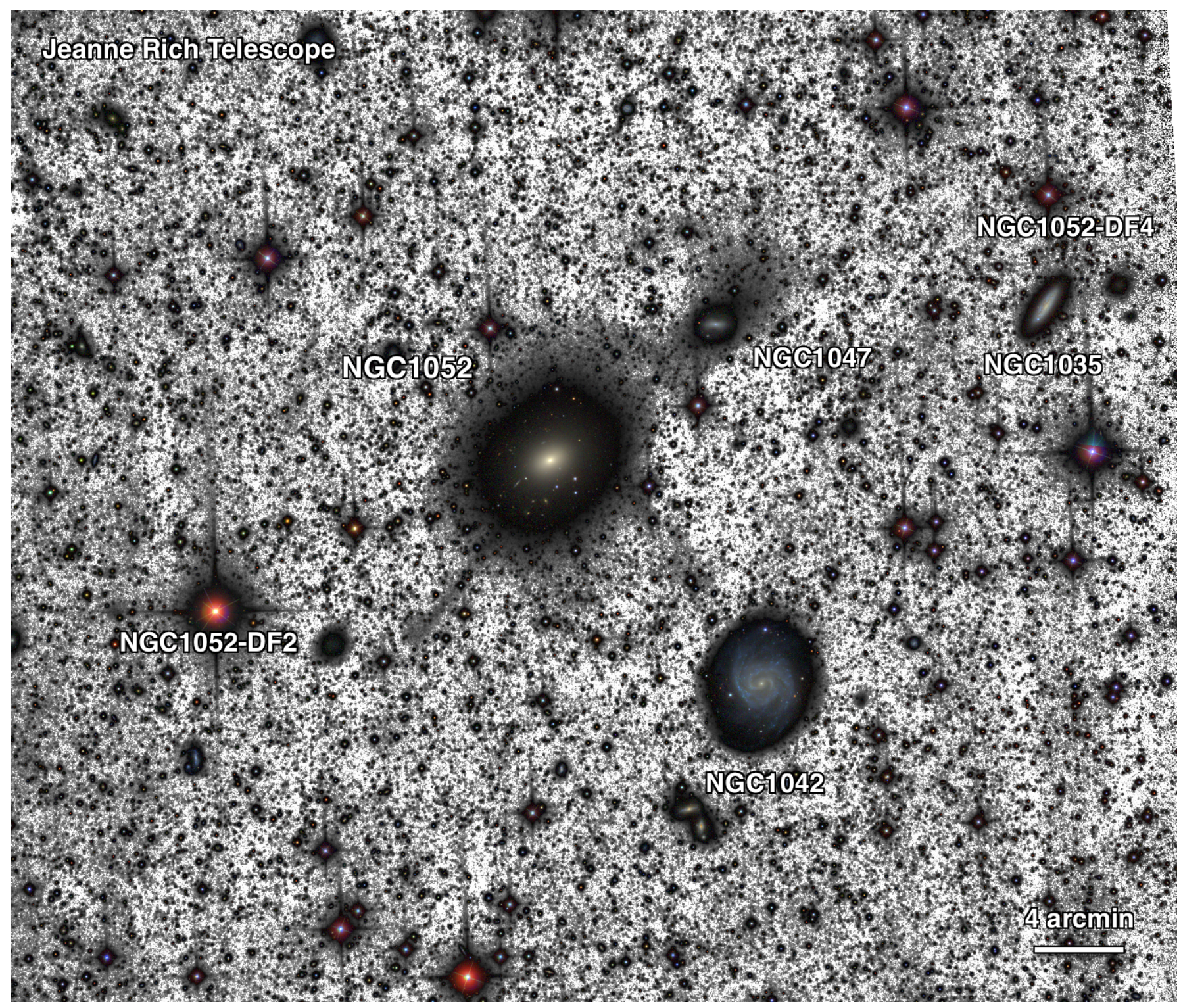

Fig. 1. NGC 1052 environment: stacked luminance $(L)$ band obtained with the Jeanne Rich telescope. True-color images from the SDSS are overlaid. The field of view is $1 \mathrm{deg}^{2}$. The indicated $4^{\prime}$ bar corresponds to $15 \mathrm{kpc}$ at $13 \mathrm{Mpc}$ or $22 \mathrm{kpc}$ at $19 \mathrm{Mpc}$. North is to the top, east to the left.

young TDG is falsified by the stellar age of NGC 1052-DF2 (9 Gyr, Fensch et al. 2019), it might be argued that it is an old TDG, formed during an interaction $9 \mathrm{Gyr}$ ago. This could solve the dark-matter-deficiency riddle and would be consistent with the location of NGC 1052-DF2 on the metallicitystellar mass relation (see discussion in Fensch et al. 2019). However, tidal features such as Stream 1 should disappear in a few gigayear, especially if the galaxy suffered posterior mergers: some of the most extended tidal arms are around $100 \mathrm{kpc}$ long, and the free-fall time from such a distance to NGC 1052 is about 1-2 Gyr. This is substantially shorter than the estimated age of NGC 1052-DF2. The linear morphology of Stream 1 is not expected if the stream had orbited the host galaxy several times. We can therefore conclude that the alignment between this stream and NGC 1052-DF2 is pure chance and is not linked with any TDG scenario. In addition, Stream 1 is not the stripped tail of NGC 1052-DF2 because if this were the case, we would expect that the tidal tail and the progenitor are connected (see, e.g., Fig. 10 of Müller et al. 2018b). The morphology of the observed tidal features does not agree with the morphology of the tidal features in the simulation by Ogiya (2018). Alternatively and perhaps more likely, Stream 1 could originate from another stripped dwarf galaxy satellite. We note an unresolved source on the axis of the stream, which is a candidate for the stripped core of its progenitor $(02: 41: 25.3,-08: 22: 11.6)$. All of these aspects suggest that this is likely a chance alignment. This is consistent with the hypothesis that the subtracted model of NGC 1052-DF2 (Fig. 3) reveals no clear signs of tidal features.

In NGC 1042, the absence of LSB features also contains valuable information. While its systemic velocity $(v=$ $1376 \mathrm{~km} \mathrm{~s}^{-1}$; Adelman-McCarthy 2009) is consistent with the velocity of the NGC 1052 group $\left(v=1425 \pm 111 \mathrm{~km} \mathrm{~s}^{-1}\right.$; van Dokkum et al. 2018a), that is, a distance of $\approx 20 \mathrm{Mpc}$, the redshift-independent distance estimators favor a closer value (13 Mpc, Theureau et al. 2007). As with NGC 1047, a visible tidal interaction would unambiguously place NGC 1042 in the NGC 1052 group. A loop lies in the direction of NGC 1042, but its morphology is rather consistent with a stream associated with another disrupted satellite orbiting NGC 1052. No clear tidal 


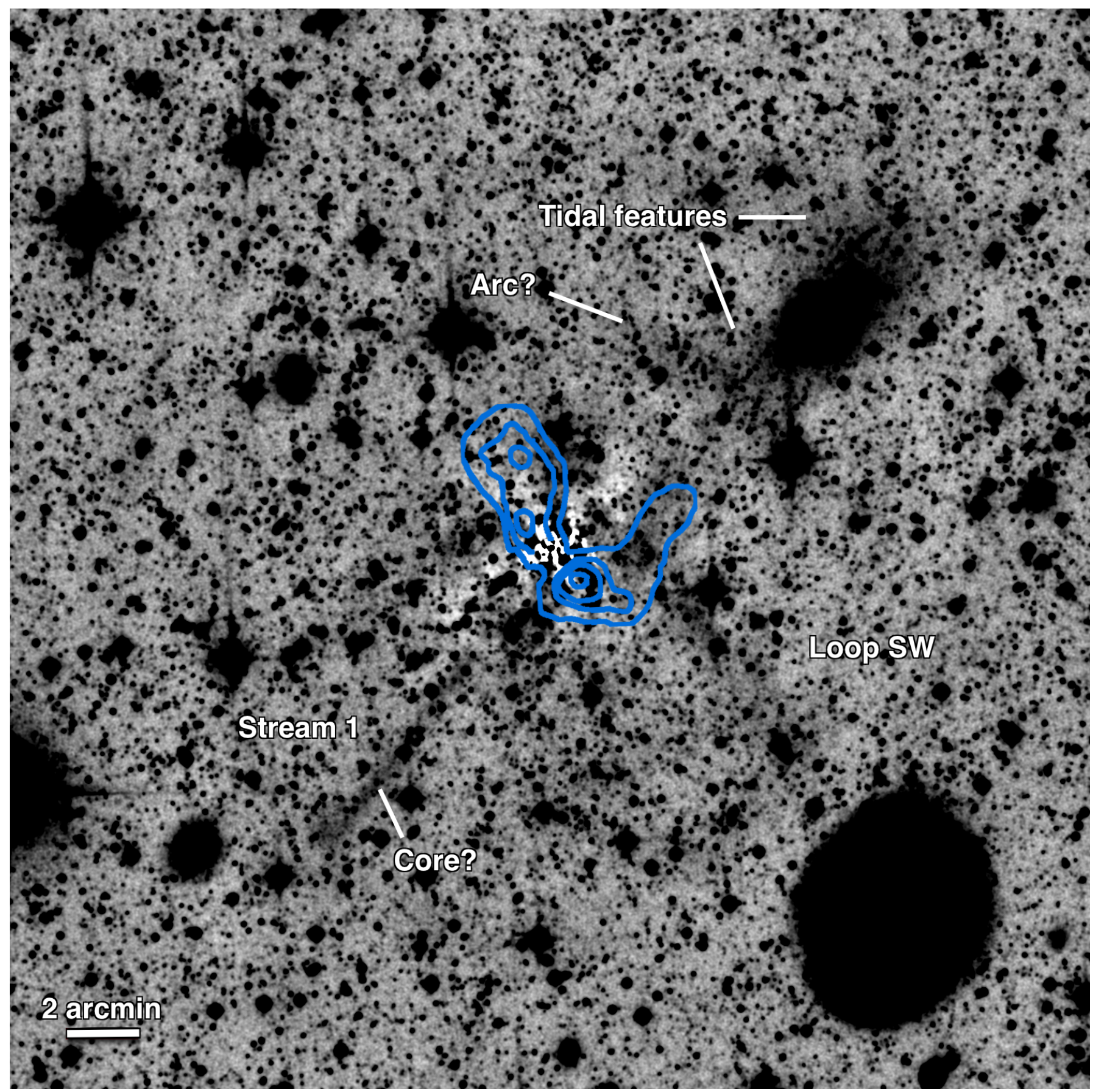

Fig. 2. Residual image after NGC 1052 was subtracted and convolved with a Gaussian kernel, revealing the LSB features in its environment. The contours show the H I distribution around NGC 1052 adopted from van Gorkom et al. (1986).

features are directly connected to NGC 1042. This can either mean that NGC 1042 is within the group or that it has just arrived in the group, and no visible interaction has yet taken place ${ }^{2}$, or that NGC 1042 is indeed only associated with NGC 1052 in projection and is, in reality, closer to us. Trujillo et al. (2019) argued that the properties of NGC 1052-DF2 and NGC 1052-DF4 would match other known dwarf galaxies if they were closer to us, for instance, at the distance of $13 \mathrm{Mpc}$. On the other hand, the spiral

\footnotetext{
2 For example, the systemic velocities for Cen A and M 83 are consistent with each other, but their separation places them more than $1.1 \mathrm{Mpc}$ away (Karachentsev et al. 2013), and no connecting LSB features have been found.
}

structure of NGC 1042 itself shows sharp changes in pitch angle and has loosely wound outer spiral arms that are quite flocculent, which is reminiscent of spirals in the early time steps of merger simulations that have not yet quite formed elongated tidal tails.

The remaining giant galaxy to be discussed in the observed field is the spiral galaxy NGC 1035. While it also has a systemic velocity consistent with the NGC 1052 group ( $v=1249 \mathrm{~km} \mathrm{~s}^{-1}$; Meyer et al. 2004), its latest distance estimate based on TullyFisher measurements is $14 \pm 2.9 \mathrm{Mpc}$ (Sorce et al. 2014). This places it in the foreground and is consistent with an absence of tidal features associated with this galaxy. 

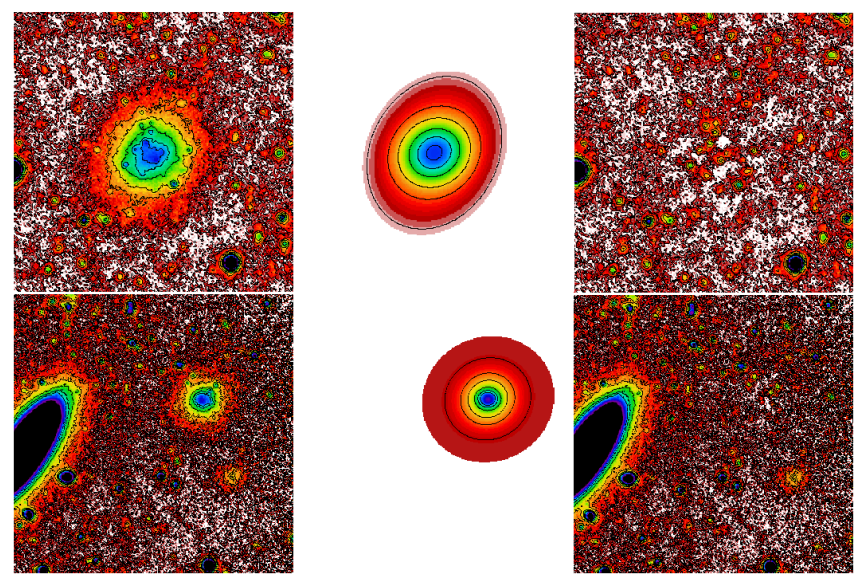

Fig. 3. Modeling of NGC 1052-DF2 (top) and NGC 1052-DF4 (bottom). Left: isophotal levels in the original image. Middle: galaxy models. Right: residual image.

\section{Conclusions}

We aimed to clarify the origin of the alleged low dark matter content of NGC 1052-DF2 and NGC 1052-DF4 by deep imaging. Deep imaging can, in principle, uncover tidal features whose morphology would reveal the origin of NGC 1052-DF2 and NGC 1052-DF4 either as tidal dwarf galaxies or a tidally dark-matter-stripped primordial dwarf. We indeed found signs of many galaxy interactions in the group. Notably, there is an ongoing tidal interaction between NGC 1052 and NGC 1047. Furthermore, around NGC 1052, we find a loop in the direction of NGC 1042 and a stream in the direction of NGC 1052$\mathrm{DF} 2$, but none of them are directly connected to these two galaxies. The features around NGC 1052 are probably much younger $(1-3 \mathrm{Gyr})$ and are inconsistent with the estimated age of NGC 1052-DF2 (9Gyr). We see no tidal features around NGC 1052-DF2 and NGC 1052-DF4, making a recent tidal stripping scenario unlikely. The tidal dwarf galaxy scenario at high redshift cannot be probed by our data because the tidal features might have been erased and may no longer be detectable today.

We further found no convincing tidal features around the spiral galaxy NGC 1042. This indicates that it is either at a larger separation within the NGC 1052 group, for example, just falling in, or that its association is only in projection and that in reality, NGC 1042 is closer to us. Their final association (physical or projected) will only be firmly established when we have accurate distance estimates to both objects.

Acknowledgements. The authors thank the referee for the timely and helpful report. The authors also thank Eric Emsellem, Anita Zanella, Remco van der Burg, Marina Rejkuba, Michael Hilker, Federico Lelli, Marcel Pawlowski, and Noam Libeskind for interesting discussions and comments. OM is grateful to the Swiss National Science Foundation for financial support. R.M.R. acknowledges the Polaris Observatory Association for hosting the Jeanne Rich telescope, Ian Kearns-Brown for supporting operations, and Parkes Whipple for assistance with data reduction. M.K.Y. acknowledges the financial support from the Scientific and Technological Research Council of Turkey (TUBITAK) under the postdoc fellowship programme 1059B191701226. I.T. acknowledges financial support from the European Union's Horizon 2020 research and innovation programme under Marie Sklodowska-Curie grant agreement No 721463 to the SUNDIAL ITN network. This research has been partly supported by the Spanish Ministry of Economy and Competitiveness (MINECO) under grants AYA2016-77237-C31-P.

\section{References}

Adelman-McCarthy, J. K. 2009, VizieR Online Data Catalog: II/294 Akhlaghi, M., \& Ichikawa, T. 2015, ApJS, 220, 1

Bertin, E. 2006, ASP Conf. Ser., 351, 112

Bournaud, F., \& Duc, P.-A. 2006, A\&A, 456, 481

Brosch, N., Kaspi, S., Niv, S., \& Manulis, I. 2015, Ap\&SS, 359, 9

Denicoló, G., Terlevich, R., Terlevich, E., et al. 2005, MNRAS, 356, 1440

Duc, P.-A. 2012, Astrophys. Space Sci. Proc., 28, 305

Duc, P.-A., Paudel, S., McDermid, R. M., et al. 2014, MNRAS, 440, 1458

Eigenthaler, P., Puzia, T. H., Taylor, M. A., et al. 2018, ApJ, 855, 142

Emsellem, E., van der Burg, R. F. J., Fensch, J., et al. 2019, A\&A, in press, DOI: 10.1051/0004-6361/201834909

Famaey, B., McGaugh, S., \& Milgrom, M. 2018, MNRAS, 480, 473

Fensch, J., van der Burg, R. F. J., Jerabkova, T., et al. 2019, A\&A, in press, DOI: 10.1051/0004-6361/201834911

Fosbury, R. A. E., Mebold, U., Goss, W. M., \& Dopita, M. A. 1978, MNRAS, 183,549

Garcia, A. M. 1993, A\&AS, 100, 47

Greco, J. P., Greene, J. E., Strauss, M. A., et al. 2018, ApJ, 857, 104

Henden, A., \& Munari, U. 2014, Contrib. Astron. Observatory Skalnate Pleso, 43, 518

Henkel, C., Javanmardi, B., Martínez-Delgado, D., Kroupa, P., \& Teuwen, K. 2017, A\&A, 603, A18

Javanmardi, B., Martinez-Delgado, D., Kroupa, P., et al. 2016, A\&A, 588, A89

Kadler, M., Kerp, J., Ros, E., et al. 2004, A\&A, 420, 467

Karachentsev, I. D., Karachentseva, V. E., Suchkov, A. A., \& Grebel, E. K. 2000, A\&AS, 145, 415

Karachentsev, I. D., Makarov, D. I., \& Kaisina, E. I. 2013, AJ, 145, 101

Karachentsev, I. D., Riepe, P., Zilch, T., et al. 2015, Astrophys. Bull., 70, 379

Kroupa, P. 2012, PASA, 29, 395

Kroupa, P., Haghi, H., Javanmardi, B., et al. 2018, Nature, 561, E4

Laporte, C. F. P., Agnello, A., \& Navarro, J. F. 2019, MNRAS, 484, 245

Luo, R., Hao, L., Blanc, G. A., et al. 2016, ApJ, 823, 85

Mancera Piña, P. E., Aguerri, J. A. L., Peletier, R. F., et al. 2019, MNRAS, 485, 1036

Martin, N. F., Collins, M. L. M., Longeard, N., \& Tollerud, E. 2018, ApJ, 859, L5

Martínez-Delgado, D., Gabany, R. J., Crawford, K., et al. 2010, AJ, 140, 962

Merritt, A., van Dokkum, P., Danieli, S., et al. 2016, ApJ, 833, 168

Meyer, M. J., Zwaan, M. A., Webster, R. L., et al. 2004, MNRAS, 350, 1195

Mihos, J. C., Durrell, P. R., Ferrarese, L., et al. 2015, ApJ, 809, L21

Müller, O., Jerjen, H., \& Binggeli, B. 2018a, A\&A, 615, A105

Müller, O., Rejkuba, M., \& Jerjen, H. 2018b, A\&A, 615, A96

Müller, O., Famaey, B., \& Zhao, H. 2019, A\&A, 623, A36

Nusser, A. 2019, MNRAS, 484, 510

Ogiya, G. 2018, MNRAS, 480, L106

Ordenes-Briceño, Y., Taylor, M. A., Puzia, T. H., et al. 2016, MNRAS, 463, 1284

Pierce, M., Brodie, J. P., Forbes, D. A., et al. 2005, MNRAS, 358, 419

Rich, R. M., Brosch, N., Bullock, J., et al. 2017, IAU Symp., 321, 186

Rich, R. M., Lee-Saunders, H., Koch, A., et al. 2018, A\&AS, submitted

Román, J., \& Trujillo, I. 2017a, MNRAS, 468, 703

Román, J., \& Trujillo, I. 2017b, MNRAS, 468, 4039

Sandage, A., \& Binggeli, B. 1984, AJ, 89, 919

Sorce, J. G., Tully, R. B., Courtois, H. M., et al. 2014, MNRAS, 444, 527

Theureau, G., Hanski, M. O., Coudreau, N., Hallet, N., \& Martin, J.-M. 2007 A\&A, 465, 71

Tingay, S. J., Edwards, P. G., \& Tzioumis, A. K. 2003, MNRAS, 346, 327

Tody, D. 1986, Proc. SPIE, 627, 733

Trujillo, I., Beasley, M. A., Borlaff, A., et al. 2019, MNRAS, in press, [arXiv: $1806.10141]$

Tully, R. B., Shaya, E. J., \& Pierce, M. J. 1992, ApJS, 80, 479

Tully, R. B., Shaya, E. J., Karachentsev, I. D., et al. 2008, ApJ, 676, 184

Tully, R. B., Courtois, H. M., Dolphin, A. E., et al. 2013, AJ, 146, 86

van der Burg, R. F. J., Muzzin, A., \& Hoekstra, H. 2016, A\&A, 590, A20

van Dokkum, P. G., Abraham, R., Merritt, A., et al. 2015, ApJ, 798, L45

van Dokkum, P., Danieli, S., Cohen, Y., et al. 2018a, Nature, 555, 629

van Dokkum, P., Danieli, S., Cohen, Y., Romanowsky, A. J., \& Conroy, C. 2018b, ApJ, 864, L18

van Dokkum, P., Danieli, S., Abraham, R., Conroy, C., \& Romanowsky, A. J. 2019, ApJ, 874, L5

van Gorkom, J. H., Knapp, G. R., Raimond, E., Faber, S. M., \& Gallagher, J. S. 1986, AJ, 91, 791

Venhola, A., Peletier, R., Laurikainen, E., et al. 2017, A\&A, 608, A142

Wittmann, C., Lisker, T., Ambachew Tilahun, L., et al. 2017, MNRAS, 470, 1512 